\title{
El cuerpo del trabajo en el capitalismo flexible: lógicas empresariales de gestión de energías y emociones
}

\author{
Maria Inés LANDA \\ Universidad Nacional de Córdoba \\ landa.mi@gmail.com \\ Leonardo Gabriel MARENGO \\ Universidad Nacional de Córdoba \\ soloporlasdudas@hotmail.com
}

Recibido: 25-8-09

Aceptado: 22-3-10

\section{RESUMEN}

Los senderos de investigación y argumentación que recorren los distintos apartados del presente artículo se interrogan en torno al estatuto del "cuerpo del trabajo" en el marco de las profundas y aceleradas mutaciones socio-estructurales que sirven de escenario a la emergencia de nuevos contextos empresariales. En función de ello, se aborda, en primer lugar, el proceso mediante el cual la categoría "cuerpo" adquiere relevancia en nuestras investigaciones en su condición de herramienta analítica. Luego, se analiza ciertos procesos de regulación de los cuerpos en espacios de implementación de nuevos dispositivos de organización empresarial. Se concluye proponiendo un conjunto de categorías que a nuestro juicio permiten delinear el complejo mapa de la empresa contemporánea en la región latinoamericana.

Palabras claves: capitalismo flexible, dispositivos de control social, empresa, trabajo, cuerpo.

\section{The body of work in flexible capitalism: Bussiness Logic of Energies and Emotions Management}

\begin{abstract}
The line of research and of argumentation that traverse the different sections of this article inquires about the status of the category "body of work" in the profound and vertiginous socio-structural changes that underlie the emergence of new business contexts. According to this, the article outlines, first, the process through which "body", as a research category, gains importance in our studies. Next, it analyses specific body regulation process in contexts where new business organization devices are implemented. Finally, it concludes proposing a set of research categories that enable to draw the complex map of contemporary business organizations in Latin America.
\end{abstract}

Key words: flexible capitalism, social control devices, business organizations, work, body. 


\section{REFERENCIA NORMALIZADA}

Landa, M.I. y Marengo, L. (2011) El cuerpo del trabajo en el capitalismo flexible: lógicas empresariales de gestión de energías y emociones”. Cuadernos de Relaciones Laborales Vol.29, Num.1, p. 177-178.

SUMARIO: Introducción. 1. Límites y posibilidades de un concepto: el "cuerpo" como categoría. 1.1. Umbrales disciplinares: una zona fronteriza entre la sociología del trabajo y la sociología del cuerpo. 1.2. Consideraciones en torno al estatuto teórico-metodológico del "cuerpo". 1.3. El cuerpo-trabajo como locus conflictual de la diagramática capitalista. 1.4. Disposiciones corporales en el capitalismo flexible. 2. La gestión de los cuerpos en la "nueva empresa". 2.1. Performances empresariales: el cuerpo como locus conflictual. 3. Consideraciones finales: El empresario de sí como emblema del management corporal. 3.1. Sistematización de categorías para el análisis del dispositivo "nueva empresa". Bibliografía.

\section{Introducción}

El presente trabajo forma parte de un programa sistemático de pesquisa e investigación (Landa, 2005; 2008; 2009a, 2009b; 2009c; 2009d; Landa y Marengo 2007a; 2007b; 2009a; 2009b; 2010a, 2010b; Marengo (2007, 2008a, 2008b, 2009) que, desde hace tres años, los autores emprendimos con el objetivo de interrogar el estatuto del "cuerpo" del trabajo en el marco de las profundas y aceleradas mutaciones socioestructurales que enmarcan la proliferación de nuevos contextos productivos empresariales en latinoamérica. En torno a esta problemática y a partir de los vectores fundamentales del nuevo capitalismo, el presente artículo expone una propuesta teórico-metodológica específica que intenta abordar críticamente las derivas del "cuerpo del trabajo" en dichas diagramáticas emergentes de interacción y reproducción social.

Las transformaciones aludidas comprometen no solo la dimensión técnicaorganizacional del proceso productivo, sino también conllevan una reconfiguración general del escenario de agentes, relaciones y procesos en el seno de las sociedades contemporáneas. Uno de los pilares sobre los cuales se edifica dicho cuadro de mutación se encuentra signado por la emergencia de los denominados dispositivos de management. En el campo de las ciencias de la administración, análisis institucional o la psicología organizacional, la noción de management, en sus distintos modelos, refiere a la disciplina específica encargada de la producción teórico-práctica de saberes y técnicas, orientados a la gestión y organización del proceso productivo y la instrumentalización de cadenas de mando y ejecución según parámetros específicos de eficiencia (Abraham, 2000; Drucker, 1996; Fernández Rodríguez, 2007a, 2007b; Linhart, 1997; Marengo, 2008b; 2009).

En el marco de dichas estrategias identificamos dos instancias fundamentales para comenzar a delinear los contornos del modelo disciplinar productivo de la nueva empresa: a) mecanismos de implicación orientados a garantizar la objetivación y la interiorización de los imperativos productivos de la empresa por parte de los empleados/ trabajadores, y b) management corporal referido a la instauración de una nueva lógica de gestión encarnada en dispositivos diversos que se sostiene en una modulación continua de las energías corporales en tanto variables destacadas de valorización y acumulación. En estos escenarios, los requisitos productivos demandan un cuadro 
renovado de competencias y aptitudes que reestructuran el modelo de inserción y metabolización de los cuerpos en la maquinaria capitalista.

El estereotipo corporal del capitalismo industrial se muestra obsoleto para abordar el embodiment sobre el cual se cimienta la empresa flexible, entendida como un campo heterogéneo y sincrético en constante desplazamiento y expansión (Couzens Hoy, 1999: 3-21). Por lo tanto, uno de los objetivos principales de la presente argumentación es avanzar en el proceso de sistematización de marcos teóricometodológicos que permitan delinear el complejo mapa de articulación e interpenetración del "cuerpo" y el "trabajo" en el capitalismo contemporáneo.

El mundo del trabajo y la producción económica históricamente han constituido objetos destacados de reflexión y análisis en el campo del pensamiento sociológico. Preguntas relativas a las lógicas de organización de la práctica productiva forjaron pautas privilegiadas para abordar la compleja trama de transición que dibuja el desarrollo del capital. Las perspectivas a partir de las cuales dicha problemática es y fue pensada, proliferan al ritmo de las constantes transformaciones de los modelos societales. De este modo, innovaciones tecnológicas, guerras, reformas y revoluciones marcan el ritmo del continuo diálogo que se produce entre teoría social y devenir histórico.

El presente artículo se ubica entre los márgenes difusos de la sociología del trabajo y la sociología de los cuerpos. Preguntarnos por las derivas del "cuerpo", nos posiciona en un terreno de difícil tránsito a causa del escaso nivel de sistematización que presenta el campo de investigación específico de lo corporal en Latinoamérica. Por lo tanto, antes de abordar el devenir del cuerpo-del-trabajo en la nueva empresa, consideramos necesario desarrollar el carácter específico que asume la categoría "cuerpo" en el marco de nuestra propuesta.

El primer apartado, analiza diversas perspectivas desde las cuales la teoría social ha intentado reflexionar en torno al "cuerpo" como objeto de investigación, para posteriormente presentar de modo sistemático el modelo teórico que constituye en líneas generales nuestra propuesta analítica "en clave corporal". En el segundo apartado, se analizan e interpretan ciertos recorridos de los cuerpos en espacios de implementación de nuevos dispositivos de organización empresarial. Por último, en el apartado destinado a las consideraciones finales, exponemos un conjunto de categorías de análisis que, a nuestro juicio, permiten comenzar a discernir la compleja trama social del dispositivo de control y gestión de la nueva empresa en Latinoamérica.

\section{Límites y posibilidades de un concepto: el "cuerpo" como categoría}

La categoría "cuerpo" se presenta esquiva, problemática y multifacética. En torno a este significante se deslizan infinidad de acepciones en el marco de las ciencias sociales y la filosofía. Su deriva presenta tantos sentidos como usos efectivos, posibles o imaginados. En ciertos planteos, la emergencia de la noción "cuerpo" podría ser reemplazada paradigmáticamente por la categoría "sujeto" o "individuo" sin comprometer demasiado el sentido. Si un concepto no refiere a cierta especificidad 
semántica rigurosa tenemos un problema. Entonces: ¿por qué hablamos de "cuerpo"?, ¿por qué no persistir en la referencia a categorías tradicionales como "sujeto" y/o "individuo"?

Por momentos, la noción "cuerpo" parecería actualizar cierta nostalgia empiricista (el cuerpo como dato "ahí en el mundo"). En otras acepciones corrientes, la categoría parecería referir a una suerte de sustancia última de lo específicamente humano (el cuerpo como sustrato natural), o también, el cuerpo como el territorio humano de lo extra discursivo (el cuerpo material). En contraste, ciertos planteos ponen el acento en el "cuerpo" como construcción simbólico-discursiva (el cuerpo-signo). Por último, encontramos derivas argumentativas en las cuales el "cuerpo" refiere a lo real-vitalinstintivo (el cuerpo potencia, el cuerpo exceso). Todas estas acepciones presentan riquezas específicas, pero también dificultades evidentes: ya sea por empiricismo ingenuo renovado, naturalismo escencialista, discursivismo imperialista o vitalismo inmanentista, en algunos casos una combinación irregular de todos estos "pecados metafísicos" dados conjuntamente. ${ }^{1}$

Más allá de que es posible y necesario someter la categoría "cuerpo" al rigor deconstructivo de la práctica crítico-analítica, existe un hecho por demás evidente: la noción de cuerpo aparece y reaparece como objeto teórico de interés (especialmente en los últimos años) en diversos espacios de reflexión y producción de conocimiento social.

Una lectura escéptica y superficial argumentará que la proliferación recurrente de este concepto, responde a un simple fenómeno de "moda", cuya emergencia se vincula a cierta vorágine banal de renovación terminológica, práctica por demás habitual en ciertos espacios académicos mercantilizados legitimados y legitimantes. Seguramente esto explica parte del problema, y nos permite desflacionar inicialmente el congestionado mercado académico de lo corporal. Algún psicoanalista argumentara, quizás con algo de verdad, que la referencia contemporánea al "cuerpo" como objeto teórico reincidente presenta un evidente rasgo sintomático, el cual se explica en determinada compulsión maquínica de sutura imaginaria (siempre fallida) ante el terror ominoso de la destitución subjetiva que trae aparejada la amenaza del cuerpo fragmentado.

A pesar de todos estos inevitables riesgos de radical diseminación, la pregunta que interpela el valor epistemológico-analítico de la categoría "cuerpo" sobrevive tanto a la envestida crítico-escéptica como a la interpretación clínico-analítica. El "cuerpo" como significante emergente e insistente del pensamiento social contemporáneo no encuentra un desarrollo sistemático, de eso no hay duda. Pero también es certero afirmar que en el marco de investigaciones y argumentaciones específicas presenta un alto potencial

${ }^{1}$ La taxonomía que aquí referimos de ninguna manera pretende constituirse en un listado completo y acabado. Su inclusión radica en que consideramos prioritario: a) destacar, que alguna manera, toda reflexión social sobre lo corporal es tributaria en cierto aspecto de estas derivas narrativas; y b) señalar el curso de las tensiones por las que naufraga el debate académico sobre el "cuerpo". 
explicativo-comprensivo. De alguna manera, "cuerpo" semantiza una dimensión de lo humano que otras categorías no han sabido o no han podido nombrar. Cuerpo significa muchas cosas, por lo tanto consideramos prioritario delimitar el alcance y la utilidad que adquiere la acepción en nuestras reflexiones.

\section{I.1. Umbrales disciplinares: una zona fronteriza entre la sociología del traba- jo y la sociología del cuerpo}

Si nos ubicamos en el arenoso terreno de las divisiones disciplinares, el presente artículo podría situarse en una especie de umbral imaginario entre la ya "consagrada" sociología del trabajo y la "emergente" sociología de los cuerpos. La categoría "cuerpo" emplazada en este umbral adquiere tonalidades específicas.

Cualquier lector habituado al campo sabe que la historia de la teorización sociológica muestra, desde sus primeros pasos, tematizaciones relativas al cuerpo y la corporalidad (Freund, 1988). El panorama se aclara inicialmente al puntualizar que lo novedoso no se encuentra en la aparición del objeto "cuerpo" en sí mismo, sino más bien en la iniciativa sistemática que intenta constituir un marco específico de reflexión teórico-sociológica en torno a lo corporal.

Los temas que interesaron a los primeros sociólogos se orientaban a explicar/comprender las características de las sociedades urbanas industriales bajo el prisma de los ejes temáticos de la época: cambio/orden social, marco económico, producción material y estructura social, entre otros. El cuerpo del "hombre", como objeto analítico, adquiere relevancia en las producciones de la sociología clásica como un elemento más de las constelaciones teóricas que pretenden explicar el conjunto de transformaciones sociales que se desplegaron en el continente europeo durante fines del siglo XIX. Mientras que la sociología del cuerpo, disciplina que emerge en escenarios de la post-segunda guerra mundial, lo que viene a introducir al interior del debate sociológico, es la mirada centrada estrictamente en la problemática del cuerpo como ámbito específico de reflexión social. ${ }^{2}$

Dicha voluntad instituyente, a la cual el presente artículo adscribe, no pretende desatender que el significante "cuerpo" desliza en una multiplicidad de acepciones que distan considerablemente de lo que podría denominarse un desarrollo homogéneo y sistemático. Como ocurre en todo universo en formación, la delimitación rigurosa de especificidades temáticas y metodológicas, se ve limitada por derivas disímiles, disputas e imprecisiones terminológicas que marcan la consistencia "gelatinosa" de

${ }^{2}$ Para profundizar en las discusiones en torno a la emergencia de la sociología del cuerpo, sus repercusiones académicas y las tensiones que introduce su constitución como enfoque disciplinar específico en el campo sociológico consultar los siguientes textos: Ahmed y Thomas (2004); Ayús Reyes y Eroza Solana (2007); Citro, (2004); Corbin, Courtine, y Vigarello, (2007) Featherstone, Hepworth y Turner (1992); Freund, (1988); Howson e Inglis (2001); Ihde (2004); Le Breton (2002); Mellor y Schilling (1997); Pedraza (2003, 2009); Schilling (1993, 1997); Soley-Beltran (2007); Turner, (1992). 
dicho campo. Apremiada por dar cuenta de las transformaciones corporales emergentes, la sociología del cuerpo decantó en una en una producción desarticulada de modelos teóricos sobre los cuerpos de los sujetos sociales contemporáneos: cuerpos discursivos, materiales, biológicos, comunicativos, medicalizados, sexualizados, disciplinados, parlantes, etc. De esta manera, a la crisis de los grandes relatos le siguió la crisis de las pequeñas historias (Grüner, 2002), que en su legítimo ánimo por ajusticiar los excesos de las totalizaciones arbitrarias de la época moderna, cayeron en un extremo tan ingenuo e inconducente como el que encarnaba su reverso. Tanto el universalismo radical como la dispersión estéril comparten en su condición de opuestos, la amenaza de entumecer la practica investigativa anulando la posibilidad de renovación, vitalidad y crítica que demanda todo hacer científico que se pretenda riguroso.

\section{I.2 Consideraciones en torno al estatuto teórico-metodológico del "cuerpo"}

Según intentamos mostrar, la pregunta por el cuerpo en la teorización contemporánea habita regiones problemáticas signadas por una marcada imprecisión y vaguedad. $^{3}$ Por lo tanto, consideramos necesario reconocer y actualizar reflexivamente (en términos bourdieanos) esta serie de limitaciones. Estamos convencidos que sería un error pretender subsanar este riesgo conjurando a modo de garante una teoría general de lo corporal que aglutine en un mismo y único espectro un universo que se muestra tan heterogéneo como fructífero. Precisamente, consideramos que la potencia y productividad de la perspectiva, radica en desplegar el amplio abanico de procesos y formas desde las cuales es posible pensar el cuerpo desde la complejidad de lo social, y viceversa.

Presentar un alegato acerca de la necesidad de impulsar y defender esta suerte de apertura disciplinar que impida una sutura última, no implica desatender la necesaria rigurosidad que es preciso adoptar para encarar una investigación sistemática de lo corporal. Pero sí reconocer que así como no existen leyes generales del devenir de los cuerpos, tampoco fórmulas universales para su abordaje. Consideramos que la manera más adecuada de transitar estas dificultades es aspirar a producir un conocimiento situado ${ }^{4}$ que en términos concretos refiere a una práctica de investigación social continuamente complementada por procesos reflexivos que inquieran sobre el contexto en el que se enmarca el estudio y la posición de enunciación del sujeto que investiga (Haraway, 1995: 313:345). Perspectiva que en última instancia apunta a una apro-

${ }^{3}$ Iniciativa que encuentra desarrollos diferenciales en distintas regiones, nuestro diagnóstico se dirige específicamente al estado de la cuestión de la disciplina en Latinoamérica, aunque no desconocemos que recibe un tratamiento más sistemático en países como Estados Unidos, Francia e Inglaterra.

${ }^{4}$ Los saberes situados teorizados por Donna Haraway son aquellos que localizan "el sujeto productor de conocimiento con todas sus marcas históricas, geoculturales e identitarias" (Belausteguigoitia: 2005:10). 
piación creativa y rigurosa del saber producido, manteniendo y habitando la tensión entre lo particular y lo general. En otras palabras, y parafraseando la reflexión wittgensteniana en torno al problema del significado, es el "uso" de la categoría cuerpo, en cadenas argumentativas específicas, quien dictaminará la potencia, la funcionalidad y la pertinencia de dicho concepto en la producción crítica de un conocimiento social.

La categoría "cuerpo" antes que constituir un reducto sustancial desde el cual delimitar un objeto definido y exhaustivo, es referida en el marco de nuestras investigaciones en su condición de tópico-analítico-específico, el cual adquiere consistencia y ponderación al integrarse al herramental teórico-metodológico que constituye, en líneas generales, el presente abordaje "en clave corporal". La pregunta ontológica general acerca de ¿qué es el cuerpo?, no es más que un falso problema. No buscamos un fundamento, sino más bien una herramienta.

\section{I.3 El cuerpo-trabajo como locus conflictual de la diagramática capitalista}

En concordancia con lo argumentado en los distintos puntos del primer apartado, intentaremos delimitar a continuación el mapa teórico a partir del cual recorremos la problemática del cuerpo-del-trabajo en el capitalismo contemporáneo. Los conceptos centrales que encauzan la dinámica analítico-argumentativa que constituyen la presente propuesta son: a) Diagrama/Dispositivo y b) Energía / cuerpo-trabajo. ${ }^{5}$

a) Diagrama /dispositivo: Referir a este concepto implica pensar los procesos de relación e interacción social en términos de geografías, geometrías y juegos corporales, en los cuales agentes y subjetividades se definen en virtud de posiciones y disposiciones corporo-energéticas específicas (Bourdieu, 1986:183-194; Foucault, 1987: 49-71). Éstas determinan agenciamientos particulares desde los cuales, en distintos niveles, es posible establecer una cartografía de procesos en el marco de espacios de constricción, producción, concentración y circulación de relaciones de poder. En estas diagramáticas relacionales, asume consistencia el concepto de dispositivo como una categoría que permite abordar el problema del poder a partir de la articulación compleja de elementos heterogéneos (Agamben, 2005; Deleuze 1990; Foucault 2006: 84). De esta manera, prácticas discursivas, prácticas no discursivas (no específicamente objetivantes), tecnologías, seres bióticos, seres a-bióticos, ${ }^{6}$ espectros y fantasmas configuran un cuadro relacional de disposiciones que marcan la dinámica de agenciamientos, funciones y procesos.

b) Energía y cuerpo-trabajo: Ambos conceptos se definen vincularmente ya que se encuentran íntimamente emparentados. La física escolar nos informa acerca de la

\footnotetext{
${ }^{5}$ Primeras aproximaciones en la elaboración y uso de dicho marco analítico en: Landa, 2005, 2008, 2009a, 2009b; Landa y Marengo; 2007a, 2007b, 2009a; 2009b; Marengo, 2009.

${ }^{6}$ Para un análisis deconstructivo de la noción de "naturaleza" y la lógica del cyborg como una resultante de la articulación compleja de elementos diversos ver: Haraway (1999: 121-163).
} 
estrecha relación que existe entre las categorías de energía, cuerpo y trabajo. En primer lugar, se advierte que la noción de energía no es pensada en términos metafísicos esencialistas. A partir de dicho concepto se intenta dar cuenta de cierto quantum, potencia o intensidad que es posible establecer como la propiedad identificable de determinados procesos. La noción de energía que aquí presentamos solo puede ser pensada en un marco relacional de vértices, posiciones y disposiciones entre los cuales se establecen movimientos, desplazamientos, transferencias, coagulaciones y capturas de dichas intensidades. En esta dinámica relacional, la circulación del referido quantum permite delinear ciertos efectos a modo de huellas, marcas y heridas sobre las geografías recorridas. Toda práctica en su condición de agenciamiento activo (ya sea progresivo o restrictivo) dibuja un entramado de "cicatrices" en los cuerpos que atraviesa.

El concepto de "energía" asume consistencia al articularse con el concepto de "cuerpo-trabajo". Partimos de los siguientes supuestos:

a) Todo acto de trabajo implica circulación de energía, sino, no es tal. Todo quantum de energía corporal puede ser potencialmente desplegado como acto laboral. Por lo tanto, referir al concepto de cuerpo-trabajo implica pensar determinadas relaciones en las cuales los cuerpos establecen acciones específicas, en el marco de disposiciones sociales, estructuras de poder (asimétricas-diferenciales) y procesos de objetivación.

b) El trabajo es pensado como la instancia (acontecimiento) y el lugar (locus) en la/el cual se comprometen las propiedades de los cuerpos humanos para producir valores sociales en su acción performativa concreta. Valor no como "medida" objetiva sino como intensidad objetivada o potencia objetivable. Hay circulación de "valor" allí donde una determinada "intensidad" proveniente de un cuerpo (individual o colectivo), se constituye en objeto de disputa en la dinámica de cierta economía del poder. En consecuencia, la variable cuerpo-trabajo, su valor, su potencia y su agenciamiento en torno a determinados recorridos del poder, solo emerge en el marco de relaciones sociales específicas, que se constituyen en un entramado reticular de escenarios, guiones, personajes y máscaras que circunscriben el devenir de toda práctica socio-productiva.

\section{I.4 Disposiciones corporales en el capitalismo flexible}

El conjunto de reflexiones que hasta aquí presentamos referidas al estatuto de la categoría cuerpo en el pensamiento social, y la perspectiva "en clave corporal" que proponemos para el estudio de las nuevas lógicas de organización y gestión de las empresas contemporáneas, es producto de una serie investigaciones empíricas que venimos realizando en torno a las especificidades del dispositivo del management en espacios productivos informacionales en Argentina (Echevarría et.al., 2007; Landa, 2008, 2009b; Landa y Marengo, 2007b; 2009a, 2009b; Marengo 2007, 2008a, 2008b).

Según dijimos más arriba, el management se define como la disciplina específica encargada de la producción e implementación de saberes y técnicas, orientados a la 
gestión, mando y organización del proceso productivo en instancias empresariales contemporáneas (Fernández Rodríguez, 2007b; Marengo, 2009). A partir de las prescripciones de estas lógicas de gestión se edifican un conjunto general de vectores ético-teórico-prácticos a partir de los cuales se estructuran las diagramáticas laborales que ubicamos bajo el rótulo "nueva empresa". En el marco de dichas estrategias, el modelo disciplinar productivo de las sociedades informacionales pone en funcionamiento múltiples tecnologías y mecanismos orientados a garantizar la objetivación y la interiorización de los imperativos productivos de la empresa por parte de los empleados/ trabajadores. Dichos mecanismos de implicación se entraman en un proceso que denominamos management corporal, entendido como una nueva lógica de gestión, encarnada en dispositivos diversos, que basa su modalidad de funcionamiento en una regulación continua de las energías corporales. La categoría "cuerpo-trabajo" adquiere centralidad al dar cuenta tanto del objeto como de la divisa que marca la lógica práctica de dicho diagrama. A continuación exponemos aquellas dimensiones relevantes y recurrentes, las cuales a nuestro juicio permiten identificar ciertos principios característicos y tendenciales de regulación y producción en estos emergentes espacios laborales.

\section{La gestión de los cuerpos en la "nueva empresa"}

La noción "nueva empresa" es referida para caracterizar un conjunto heterogéneo de organizaciones y diagramáticas de interacción social que encuentran su particularidad en ciertos parámetros funcionales característicos del capitalismo tardo-moderno. Relacionar dicha organización con el modo de producción histórico que la engendra no implica desconocer sus especificidades funcionales y estructurales, pero consideramos prioritario destacar no solo su carácter de acontecimiento diferencial sino también el principio de continuidad que liga dichos espacios con la lógica del beneficio, acumulación y explotación capitalista (Boltanski y Chiapello, 2002; Marengo, 2009).

Somos plenamente conscientes que al unificar un conjunto tan diverso y amplio de espacios a partir de una categoría general, se ejerce, en mayor o menor medida, algún grado de "violencia" sobre las particularidades de cada unidad del denominado conjunto. Optamos por emprender esta suerte de simplificación del abanico de organizaciones que se articulan en torno a la categoría "nueva empresa" priorizando el valor expositivo.

${ }^{7}$ Por otra parte, cabe resaltar que dichos principios, un tanto genéricos, se desprenden de un conjunto de investigaciones de casos, en diversos ámbitos empresariales. Para profundizar en torno al uso del concepto nueva empresa y management: a) en Call Centers ver: Echeverría, et.al. (2007); Landa y Marengo (2007b); Marengo (2007; 2008a; 2008b); b) en Fitness Centers consultar: (Landa, 2008; 2009a; 2009b; 2009c); c) la articulación entre espacios empresa- 
La "nueva empresa" es una entidad con fines lucrativos integrada por capital y trabajo como factores privilegiados de producción (Wittke, 2006). El trabajadorempleado y la empresa-capital establecen una relación de intercambio, que en una dimensión superficial-descriptiva, se sustenta en una transacción de un monto de dinero-salario por tiempo de trabajo, en tanto energía productiva humana objetivada en un "hacer" valorado.

Hasta el momento, las características a partir de las cuales definimos a la "nueva empresa" no marcan ninguna diferencia sustancial con la unidad productiva del capitalismo histórico. La propiedad privada de los medios de producción, la circulación e intercambio de mercancías y la explotación del trabajo humano constituyen parámetros que permiten identificar evidentes líneas de continuidad entre las actuales configuraciones productivas y la lógica instrumental del capital. Las continuidades encuentran su realización en cuestiones relativas tanto a disposiciones sociales, marcos político-institucionales, estructuras de poder, dispositivos técnicos y soportes ideológicos concretos (Boltanski y Chiapello, 2002, Marengo, 2008a, 2008b). El capitalismo sigue encontrando en la articulación compleja entre energía, trabajo, cuerpo y capital su instancia privilegiada de realización (Landa y Marengo, 2007a; 2007b; Landa y Sorribas, 2006; Marengo, 2009).

Uno de los rasgos excluyentes que caracterizan esta lógica de producción social, y que compete a la incorporación de los cuerpos humanos al sistema de producción capitalista, es la modalidad de metabolización a partir de la cual el sistema productivo asimila la figura del trabajador (Métszáros, 1995: 117). Un cuerpo debe actualizar ciertos principios definidos para acceder al estatuto de cuerpo-trabajo. Los parámetros que determinan el tamiz del dispositivo disciplinar capitalista se dirimen principalmente en la dupla: productividad económica y docilidad política. ${ }^{8}$ Según especificamos, la lógica del beneficio como principio formal característico de la continuidad capitalista trasciende las particularidades de las distintas actualizaciones de dicho modo de producción. La demanda de trabajadores dóciles y productivos, se presenta como una constante histórica en aquella lógica de producción y acumulación. Pero los parámetros de delimitación, semantización y performatización de la productividad económica y la docilidad política del factor trabajo, se actualizan de acuerdo a transformaciones históricas determinadas, las cuales comprometen la emergencia e incorporación al mundo de la producción de saberes, técnicas y prácticas específicas que reconfiguran el cuadro de agentes y procesos.

riales (tales como los Call Center con los Fitness Centers) consultar: Landa y Marengo (2007a; 2009a; 2009b; 2010b).

${ }^{8}$ Según lo desarrolló con elocuencia Michel Foucault en el primer tomo de Historia de la Sexualidad (2003: 168). No obstante, es preciso remarcar que Foucault utiliza alternadamente la noción de utilidad y de productividad, en articulación con el concepto de docilidad. La referencia a uno u otro concepto acentúa diferencialmente las vinculaciones entre la esfera económica y la específicamente política. Para profundizar en torno a esta relación ver: Foucault (2002 y 2003:147) y Gaudemar (1998). 
En estos nuevos espacios empresariales se está produciendo una redefinición de las aptitudes y competencias que determinan la incorporación activa del cuerpotrabajo al proceso productivo. La disposición corporal del trabajador fabril, la cual demandaba músculos tonificados y predisposición mecánica, en ciertos contextos, cede su lugar hegemónico a un modelo de trabajador al cual se le exige el embodiment integral de competencias comunicativas, afectivas y emocionales (Castellanos Ortega y Pedreño Cánovas, 2006; Coriat, 1993;; Echevarria et.al., 2007; Landa y Marengo, 2007a, 2007b; Marengo, 2009; Virno, 2008). Aunque las lógicas de producción en la nueva empresa presenten rasgos evidentes de continuidad con el círculo del fordismo (Sennet, 1998: 45), en términos de rituales maquínicos de desempeño, la modalidad de metabolización del trabajo en estos contextos parecería mostrar un diagrama de funcionamiento y acumulación que excede y desafía los esquemas clásicos de abordaje del mundo del trabajo en el capitalismo.

Lejos quedó la disposición corporal fabril. Hoy las demandas corporales parecen ser otras. En este panorama, palancas, poleas y sistemas hidráulicos ceden su protagonismo ante pantallas, ordenadores, headsets y sonrisas telefónicas (Landa y Marengo, 2007b). Dichos escenarios de innovación tecnológica constituyen un terreno privilegiado para identificar lineamientos y preguntas generales acerca del modo a partir del cual se están incorporando los agentes en la producción capitalista tardomoderna (Del Bono, 2002, Landa, 2009c; Marengo, 2008b).

Reconocer que la performatización del cuerpo-trabajo sufre transformaciones radicales en el capitalismo contemporáneo no implica desatender que, como señala Manuel Castells (2002:309):

“...nunca fue el trabajo más central en el proceso de creación de valor. Pero nunca fueron los trabajadores, (prescindiendo de su cualificación) más vulnerables, ya que se han convertido en individuos aislados sub-contratados en una red flexible, cuyo horizonte es desconocido incluso para la misma red".

A pesar de su carácter fundamental, este desplazamiento no genera una homogenización de los trabajadores en torno al modelo fantaseado de trabajador cognitivo, según parecerían definirlo tanto el post-industrialismo ${ }^{9}$ como ciertos planteos del autonomismo italiano. Según nuestra perspectiva, el diagnóstico más riguroso es aquel que identifica como rasgo característico del mundo del trabajo contemporáneo, una progresiva polarización en la estructura ocupacional en la que se produce una partición entre trabajadores genéricos reemplazables precarizados e inscriptos aún en el círculo del fordismo y una elite ilustrada de trabajadores informacionales propiamente dichos, los cuales constituyen una minoría privilegiada tributaria de valores como la inventiva, imaginación, autonomía y autogestión (Castells, 2002: 234; Landa y Marengo, 2007b).

\footnotetext{
${ }^{9}$ Para profundizar en la crítica de los diagnósticos post-industrialistas ver: Castells, (2002: 234).
} 
A pesar de las experiencias diferenciales que encuentra cada uno de estos modelos-tipo de trabajador en su práctica laboral, existen principios generales de estructuración de la producción informacional que afectan al conjunto. A nivel macro y micro estructural, el paradigma de la flexibilidad se configura como un principio axiomatizador de la actual lógica empresarial (Guattari, 2004: 58-64). Como ya anticipamos, en torno a este significante se estructuran distintos procesos y mecanismos organizacionales que se traducen en la implementación de dispositivos sociotécnicos diversos. La constitución de un régimen de disciplina relativamente sistematizado que permita encauzar la capacidad laboral de los cuerpos humanos en un esquema colectivo de producción y acumulación aparece como un principio recurrente de las prácticas de management.

En el marco de dicha lógica, el cuerpo económicamente productivo ya no es el cuerpo mudo, robotizado y muscularmente tonificado del fordismo. El paradigma de la "flexibilidad" como requisito contemporáneo de empleabilidad en el mundo productivo capitalista, demanda actualizaciones de aquel modelo fabril burocráticodisciplinar.

La nueva economía del poder en la empresa contemporánea parece asumir modalidades de funcionamiento cercanas a lo que Deleuze (1998: 105) denominó dispositivos de control, o el mismo Foucault (2006: 84) estableció como lógicas de seguridad, signadas ya no en el horizonte de la normación disciplinar sino bajo el paradigma de normalización biopolítica. Aspectos tales como autonomía, auto-gestión, polivalencia, liderazgo, grupalidad y creatividad constituyen hoy los parámetros de inclusión por excelencia del universo productivo de la "nueva empresa".

La presencia de esta serie de atributos corporales entre los operarios de una planta productiva de la década del 50', hubieran significado altos niveles de "peligrosidad" bajo la lupa de un ingeniero industrial fordista. Pero en el actual modelo informacional de la empresa, el despliegue integral de las competencias cognitivas, afectivas, emocionales y comunicacionales de los cuerpos constituye un insumo de alto valor. Por lo tanto, nos ubicamos en un momento en el cual la demanda de rentabilidad provoca actualizaciones de lo políticamente conveniente en términos de control del proceso productivo.

En este sentido, la garantía de docilidad política debe realizarse en sinergia positiva con estos requisitos de productividad flexible. Esta demanda se inscribe a partir de la implementación de múltiples dispositivos que articulan modalidades clásicas de disciplinamiento corporal con nuevas lógicas de gestión, modulación y captura de energías corporales. La consistencia gaseosa de la nueva empresa flexible (Deleuze, 1998:102), debe inventar continuamente estrategias de control y organización que aseguren los requisitos de rentabilidad sin cortar la circulación de flujos productivos. De esta manera, la práctica managerial ha renunciado progresivamente a ser una ciencia metódica de pretensiones exactas para apuntar a constituirse en un verdadero arte (Abraham, 2000:35). 


\subsection{Performances empresariales: el cuerpo como locus conflictual}

Tal como planteamos, uno de los elementos a partir de los cuales abordamos la problemática del cuerpo-trabajo gira en torno a los procesos de objetivación y producción de valores sociales. Según lo puntualizara Marx (1973: 29), en el marco de la producción simple de mercancías, la dinámica de explotación, valorización y acumulación capitalista presenta un esquema bastante claro en el que el trabajo humano es "objetivado" en la materialidad de la mercancía, funcionando como el "combustible" privilegiado de la reproducción del capital. Existen infinidad de discusiones en torno a la lógica última del proceso de acumulación, valorización y reproducción que no consideramos pertinente actualizar en este escrito. Preferimos centrarnos en la dinámica de "objetivación" la cual parecería dar cuenta de cierto rasgo general de todo proceso de trabajo humano, inclusive el denominado trabajo inmaterial. De esta manera, identificamos como el rasgo sobresaliente del trabajo en el marco del capitalismo contemporáneo la configuración del mismo en tanto "hacer valorado" en el mercado productivo. Dicha operación analítica nos permite operar con la categoría de "valor trabajo" por fuera de la encallada discusión de la teoría clásica marxista referida a la mensurabilidad de la mercancía que ve en la teoría del valor una teoría de medida (Cutler et. al., 1977).

Según Andre Gorz (2003:48) en la emergente lógica de producción empresarial dicho esquema de objetivación y acumulación se complejiza de tal manera que produce una instrumentalización integral del cuerpo en la cual la empresa compra, ante todo, a la persona y su devoción. Es decir, el principio de funcionamiento ya no se realizaría en la mera incorporación del trabajo humano a partir de la mediación de su potencia objetivada en una mercancía, sino en un "darse en cuerpo y alma" de estos sujetos trabajadores. El proceso de trabajo encuentra su "objetivación" en el propio cuerpo en tanto locus conflictual de realización. El resultado no se presenta como una "externalidad-objeto-mercancía", sino más bien, decanta en una performance corporal holística. En la producción clásica de mercancías, los trabajadores no "pertenecían" a la empresa, el vínculo radicaba en un modelo de trabajo definido como acción meramente instrumental en el cual no se demandaba un compromiso integral subjetivo (implicación) con la actividad, sino más bien un intercambio (coaccionado, reglado y necesariamente desigual) en el que los trabajadores "prestaban su cuerpo" al cumplimiento de tareas cuyo fin no tenían necesidad de convalidar o conocer. El trabajador ingresaba así al proceso de explotación capitalista desde cierta exterioridad; no era necesario "creer", lo prioritario era "hacer". Precisamente en el carácter constitutivo de dicho vínculo de alteridad, de la posibilidad de diferencia entre la esfera de la práctica y la creencia, basaron sus esperanzas gran parte de los discursos políticos revolucionarios de la modernidad, entre ellos, como figura privilegiada el marxismo. En dicho escenario, el compromiso subjetivo del trabajador necesariamente acentuaba la eficacia disciplinar productiva, pero no era más que un componente accesorio.

Siguiendo a Gorz (1998: 48), en el actual esquema empresarial se observa una tendencia a dejar progresivamente el plano del trabajo abstracto para volver a la "prestación personalizada", lo cual despierta un cúmulo de interrogantes no solo sociológicos sino también fundamentalmente políticos, ya que esta mutación de 
lógicas y procesos redefinen los vínculos entre el capital y el trabajo, inaugurando un nuevo universo simbólico, ideológico y comunitario en el interior de los espacios productivos contemporáneos.

\section{Consideraciones finales: El empresario de sí como emblema del management corporal}

Uno de los aspectos más novedosos del cuerpo-trabajo del informacionalismo (Castells, 2002: 256-257) es que no se constituye a partir de parámetros de normalidad en referencia a un "molde" prefijado de ritmos y competencias técnicas. Hoy el paradigma de trabajador-empleado es aquel que se constituye en empresario de sí mismo a partir de una adopción devota de los parámetros éticos y de los objetivos instrumentales de la empresa en su condición de comunidad imaginada (Castellanos Ortega y Pedreño Cánovas, 2006: 71-106; Foucault, 2007:263-264, Du Gay, 2003: 251-280). Esta modalidad de sujeción, principalmente asentada en componentes afectivos y emocionales, se muestra más adecuada para resistir los embates de la "renovación continúa de las instituciones" en el vertiginoso capitalismo flexible (Sennett, 1998: 51). La empresa interpela al individuo a priorizar por sobre cualquier referencia identitaria externa (clase, religión, etnia, etc.) su adscripción al universo vincular ético-imaginario de la "marca" en su condición de entidad espectral tendencialmente vacía.

Es el cuerpo mismo y la suma de sus competencias integrales la mercancía de alto valor que es metabolizada en el proceso de acumulación de la nueva empresa. Precisamente, nuestro foco de interés se concentra en torno al estatuto que asume el cuerpo-trabajo como cuasi-totalidad (suma de competencias cognitivas, afectivas, emocionales y comunicacionales) en el diagrama de acoplamiento productivo empresarial.

Esta lógica de incorporación encuentra su correlato específico en una serie de dispositivos técnicos orientados tanto a la potenciación de las aptitudes y competencias del denominado estereotipo corporal de trabajador empresarial-modelo (management corporal), como así también al diseño e implementación de lógicas de control y disciplinamiento que garanticen la adecuación de la práctica productiva individual y grupal a los imperativos generales de la empresa (mecanismos de implicación).

En estos recorridos corporales las pretensiones del dispositivo nueva empresa, según se vienen desarrollando, no siempre se manifiestan en una performatización precisa que actualice a rajatabla la corporalidad prescripta. A partir de una primera aproximación al campo podemos identificar ciertos rasgos específicos en la intersección entre este dispositivo socio-técnico-productivo y los escenarios y corporalidades locales. La nueva empresa latinoamericana a pesar de embanderarse en torno a la ideología del management, asume determinadas configuraciones que se actualizan en relación directa con el contexto puntual en que estas técnicas y disposiciones se apliquen. Precisamente, la capacidad adaptativa de la gestión a condiciones cambiantes y contingentes es uno de los principios fundantes de este paradigma. Por lo tanto, no se trata de realizar diagnósticos y modelos teóricos últimos y completos que captu- 
ren al detalle la heterogeneidad de las diversas experiencias y manifestaciones; más bien de lo que se trata es de elaborar herramientas teóricas e investigaciones empíricas que presenten tanta elasticidad y amplitud como el universo cambiante y escurridizo que se pretende estudiar. En consecuencia, concluimos esta disertación proponiendo un conjunto de categorías que a nuestro juicio permiten comenzar a delinear el complejo mapa de la empresa contemporánea.

\subsection{Sistematización de categorías para el análisis del dispositivo "nueva em- presa"}

Según desarrollamos, referir a la noción de "dispositivo" para abordar y caracterizar el universo de la nueva empresa, implica pensar en directa articulación una serie compleja de elementos heterogéneos. Por ello, en el marco propuesto se entretejen en torno a este concepto imbricaciones entre tecnologías, cuerpos, prácticas, saberes, discursos y sujetos que delimitan los recorridos del poder y las disposiciones sociales en un cuadro diagramático particular.

A partir de nuestras investigaciones destacamos del cuadro general de categorías analíticas utilizadas un conjunto de dimensiones específicas, ${ }^{10}$ las cuales son susceptibles de ser abordadas individualmente o en articulación con otras. A continuación presentamos aquellas que consideramos más relevantes y fructíferas para comenzar a sistematizar el complejo universo del "dispositivo nueva empresa".

- Tecnologías de escaneo y selección de cuerpos de alto valor: Mediante un conjunto de prácticas específicas el dispositivo del management configura el cuadro de agentes y procesos según el esquema de polarización de los cuerpos-trabajo anteriormente descripto. Cabe resaltar, que no existen $a$ prioris estrictos mediante los cuales la empresa seleccione a sus recursos humanos de alto valor. Ésta ópera pragmáticamente en busca de individuos que reúnan aptitudes potenciales para desempeñarse como cuerpos-líderes. La selección de dichos cuerpos, se realiza en la dinámica cotidiana de interacción. De este modo, en un proceso de retroalimentación constante la empresa mide "sobre la marcha" el desempeño individual de cada cuerpotrabajo y su grado de correspondencia según las demandas de docilidad política y productividad económica prescripto por la literatura managerial y enunciado en los objetivos instrumentales del departamento de gestión (Landa y Marengo, 2007b; 2010b).

- Tecnologías de potenciación de competencias afectivo-emocionales: Según se puntualizó, el modelo de acumulación, explotación y control de la

\footnotetext{
${ }^{10}$ Para acceder a experiencias de aplicación concreta de estas categorías y dimensiones en contextos empresariales ver: Echevarría, et. Al (2007); Landa (2008, 2009a; 2009b; 2009c); Marengo y Landa (2007a, 2007b, 2009a; 2009b; 2010b), y Marengo (2007, 2008a, 2008b).
} 
nueva empresa se asienta en la captura de competencias afectivo-emocionales en tanto instancias privilegiadas de valorización. Dicha prerrogativa se traduce en la instrumentalización de técnicas que apuntan a maximizar la secuencia de emergencia y rendimiento de aptitudes ponderadas positivamente en el proceso de trabajo. Por lo tanto, la identificación de variables de maximización de tales atributos, y el diseño de estrategias de operación sobre los mismos impacta directamente en las curvas de productividad (Landa y Marengo; 2007b, 2009b).

- Mecanismos de regulación/compensación de desgaste y habituación de competencias corporales: El "darse en cuerpo y alma" del trabajador en el esquema empresarial contemporáneo y la demanda de compromiso integral de competencias físico-psíquico-comunicativas introduce a estos cuerpos en curvas de desgaste y habituación que presentan rasgos específicos. Una tarea fundamental del dispositivo del management radica en identificar en un cuadro de variables operables el conjunto de factores que funcionan como condicionantes del rendimiento individual, grupal y colectivo. Este repertorio incluye tanto dimensiones positivas como prescriptivas en un cuadro que contempla aspectos tan heterogéneos como la organización del espacio, la combinación de colores, la elección de la música ambiental, la gestión de eventos de esparcimiento extra-institucional, e inclusive la intimidación y la amenaza (Landa y Marengo, 2007, b; 2009a).

- Practicas discursivas y no discursivas de control y disciplinamiento del cuerpo-trabajo: La concreción del esquema de docilidad política del colectivo trabajador y de maximización del rendimiento por unidad de producción demanda, dispositivos de control flexibles; es decir, que no entren en conflicto con los objetivos productivos generales. En contextos de "renovación continua" es necesario algún tipo de continente identitario común al colectivo de trabajadores que sirva de soporte a las relaciones de interacción y correspondencia entre empleados y empresa otorgando continuidad y unidad al vínculo. En el marco de las prácticas de management se contemplan mecanismos relativos a la comunicación institucional, la imagen corporativa y la comunidad empresarial que apuntan precisamente a constituir un universo ético-simbólico-imaginario hacia adentro del espacio productivo que haga de cemento social del conglomerado de trabajadores. El fomento de esta "comunidad imaginada" en torno a la figura dominante de la empresa se sustenta tanto en necesidades productivas propiamente dichas $(\mathrm{Ej}$.: grupos de trabajo), como así también en aspectos relativos específicamente al control y disciplinamiento del colectivo de trabajadores (Landa, 2009c; Landa y Marengo, 2007b; Marengo, 2008a; 2008b).

- Tecnologías de identificación y tratamiento de los cuerpos "peligrosos" y "factores contaminantes": En su diario recorrido por la cartografía corporal 
del conglomerado de trabajadores, el dispositivo de gestión "nueva empresa" no solo se concentra en variables productivas propiamente dichas, sino también en elementos restrictivos o limitantes del rendimiento general vinculados al desempeño de los recursos humanos. En el marco de dicha tarea, surge como imperativo la identificación de "cuerpos peligrosos" y "factores contaminantes". En torno a estos "males" se agrupa cualquier iniciativa de organización colectiva del tipo gremial o sectorial, y en general todo tipo de asociación que no se sostenga en los canales formales e informales que promulga la empresa. A partir de diagnósticos diariamente actualizados se seleccionan medidas específicas para compensar o suprimir las variables de riesgo (Landa y Marengo; 2009a y 2010b).

- Factores jurídico-institucionales de regulación y facilitación: El campo de operaciones de gestión empresarial se encuentra directamente limitado por el marco político legislativo de la práctica laboral vigente en cada territorio. De esta manera, es posible observar modalidades diversas de actualización del dispositivo "nueva empresa" en relación directa con el cuadro legal de regulación del mundo del trabajo y la producción. El Estado, lejos de ser un agente menor o secundario, diagrama en gran medida el campo de posibilidades de acción relativo a prácticas de management a partir de la promulgación de marcos legales e institucionales que regulen, facilitan o limitan las lógicas de gestión (Echeverría et al., 2007).

- Practicas de negociación, resistencia y soportabilidad del cuerpo-trabajo (acciones individuales y colectivas): las dinámicas de vinculación entre los trabajadores y la empresa se presentan como otra dimensión significativa del universo político institucional de la nueva empresa. Ya sea de modo individual, grupal o colectivo los trabajadores no constituyen agentes pasivos sobre los cuales se implementen lógicas de gestión sin que se registren consecuencias determinadas. Existen variables territoriales, generacionales, políticas y culturales que determinan posiciones y actitudes diferenciales por parte de los trabajadores frente al conjunto de medidas en el marco de prácticas de management (Echeverría et al., 2007; Landa y Marengo, 2007b).

\section{Bibliografía}

Abraham, T. (2000). La empresa del vivir. Buenos Aires: Editorial Sudamericana.

Agamben, Giorgio (2005). ¿Qué es un dispositivo? Conferencia ofrecida en el marco de su reconocimiento como Dr. Honoris Causa de la UNLP. La Plata.Argentina. Disponible en:

http://caosmosis.acracia.net/wp2pdf/texto_de_caosmosis.pdf

Ahmed, J. y Thomas, H. (2004). Cultural Bodies. Oxford: Blackwell Publishing Ltd. 
Antúnes, R. (2005). Los sentidos del trabajo. Ensayo sobre la afirmación y la negación del trabajo. Buenos. Aires: Herramienta y Taller de Estudios Laborales.

Ayús Reyes, R. y Eroza Solana, E. (2007). El cuerpo y las ciencias sociales. Revista Pueblos y Fronteras. Disponible en: $\mathrm{http} / / /$ redalyc.uaemex.mx/redalyc/src/inicio/ArtPdfRed.jsp?iCve=90600403.

Belausteguigoitia, M. y Leñero M. (ed.) Fronteras y Cruces: para una cartografía de escenarios culturales latinoamericanos. México: UNAM, PUEG.

Boltanski, L. y Chiapello, E. (2002). El nuevo espíritu del capitalismo. Akal: Madrid.

Bourdieu, P. (1986). Notas provisionales sobre la percepción del cuerpo. En C. Wright Mills et.al. Materiales de la sociología crítica (pp. 183-194). Madrid: La Piqueta.

Bourdieu, P. y Wacquant L. (2005). Una invitación a la Sociología Reflexiva. Buenos Aires: SXXI.

Castellanos Ortega, Mari Luz y Pedreño Cánovas, Andrés (2006). Los nuevos braceros del ocio: Sonrisas, cuerpos flexibles identidad de empresa en el sector turístico. Buenos Aires: Miño y Dávila.

Castells M. (2002). La era de la información: Economía, sociedad y cultura. Volumen 1. La sociedad red. Buenos Aires: Siglo XXI.

Citro, S. (2004). "La Construcción de una Antropología del Cuerpo". Ponencia presentada en VII Congreso Argentino de Antropología Social, Simposio Antropología del Cuerpo, Facultad de Filosofía y Humanidades-UNC, Villa Giardino, Argentina. Publicación en Actas de Congreso (Formato CD).

Colectivo Situaciones (2006). ¿Quién habla? Buenos Aires: Editorial Tinta Limón.

Corbin, A., Courtine, J. y Vigarello, G. (2007). Las mutaciones de la mirada: el siglo $X X$. Historia del cuerpo. Volumen 3. España: Taurus Historia.

Coriat, B. (1993). El taller y el robot. Ensayos sobre el fordismo y la producción en masa en la era electrónica. Madrid: Siglo XXI.

Couzens Hoy, D. (1999). Critical resistance: Foucault and Bourdieu. En G. Weiss y H. Haber (eds.) Perspectivas on embodiment. The intersections of Nature and Culture (pp. 3-21). Nueva York: Routledge.

Crisorio, R. (1998). "Constructivismo, cuerpo y lenguaje". Revista de Educación Física \& Ciencia. Núm. 4, $75-81$.

Cutler, A., Hindess, B, Hirst, P., Hussain, A. (1977). Marx capital and capitalismtoday. Londres: Routledge \& Kegan Paul. 
Del Bono, A. (2002). Telefónica. Trabajo degradado en la era de la información. Madrid: Miño y Dávila.

Deleuze, G. (1987) Foucault. Buenos Aires: Paidos.

Deleuze, G. (1990). “¿Qué es un dispositivo?”. En E. Balbier et al. Michel Foucault, filósofo. Barcelona: Gedisa.

Deleuze, G. (1998). "Postdata sobre las sociedades sin control”. En C. Ferrer (ed.) El lenguaje libertario. Buenos Aires: Editorial Altamira.

Drucker, P. (1996). La administración en una época de grandes cambios. Buenos Aires: Sudamericana.

Du Gay, P. (2003). Organización de la identidad. Gobierno empresarial y gestión pública. En: S. Hall y P. Du Gay (ed.), Cuestiones de la identidad cultural (pp. 251-280). Buenos Aires: Amorrtou.

Echevarría, L.; De Elejalde, G. y Marengo, L. (2007). (Des) conectados en tiempo real. El trabajo y su representación en los Call Centers. Un estudio de caso en la empresa Movistar". Tesis de Licenciatura en Ciencias de la Comunicación e Información. Universidad Nacional de Córdoba, Argentina. Inédito.

Featherstone, M., Hepworth, M. y Turner,B.S. (1992). The Body. Social Process and Cultural Theory. Londres: Sage Publications.

Fernández Rodríguez, J. M. (2007a). Postmodernidad y Teoría crítica de la empresa: una presentación de los Critical Management Studies. En J.M. Fernández Rodríguez Vigilar y Organizar. Una introducción a los Critical ManagementStudies. Madrid: Siglo XXI.

Fernández Rodríguez, J. M. (2007b). El discurso del management: tiempo y narración. Madrid: Centro de Investigaciones sociológicas (CIS).

Foucault, M. (2002). Vigilar y castigar. Buenos Aires: SXXI.

Foucault, M. (2003). "La verdad y las formas juridicas”, Gedisa, España.

Foucault, M. (2006). Seguridad, Territorio, Población. Buenos Aires: Fondo de Cultura.

Foucault, M. (2007). El Nacimiento de la biopolítica. Buenos Aires: Fondo de cultura Económica.

Freund, P. (1988) Bringing society into the body. En Theory and Society, 17, 839-864.

Gaudemar, J.P. (1998). Preliminares Para una Genealogía de las Formas de Disciplina en el Proceso Capitalista del Trabajo. En M. Foucault et. al. Espacios de poder. Madrid: La Piqueta. 
Gorz, A. (2003). Miserias Del Presente, Riquezas De Lo Posible. Buenos Aires: Paidos.

Grüner, E. (2002). "El fin de las pequeñas historias. De los estudios culturales al retorno imposible de lo trágico". Buenos Aires: Paidos.

Guattari, F. (2004). Plan Sobre el Planeta: capitalismo mundial integrado y revoluciones moleculares. Madrid: Traficantes de Sueños

Haraway, D. (1995). "Conocimientos situados: La cuestión científica en el feminismo y el privilegio de la perspectiva parcial “. En Ciencia, cyborgs y mujeres: la reinvención de la naturaleza (pp 313-345). Madrid: Ediciones Cátedra.

Haraway, D. (1999). Las promesas de los monstruos: Una política regeneradora para otros inapropiados/bles. Politica y Sociedad, 30, 121-163.

Howson, A. e Inglis, D. (2001). The body in sociology: tension inside and outside sociological thought. Sociological Review, 49 (3), 297-317.

Ihde, D. (2004). Los cuerpos en la tecnología. Nuevas tecnologías: nuevas ideas acerca de nuestro cuerpo. Barcelona: Editorial UOC.

Landa, M.I. (2005). "Diagrama, poder y tecnologías: un modo de pensar las prácticas corporales desde Foucault". Ponencia presentada en el Sexto Congreso Argentino y Primero Latinoamericano de Educación Física y Ciencias. La Plata: Facultad de Humanidades y Ciencias de la Educación.Universidad Nacional de La Plata. Inédito.

Landa, M.I. (2007). Experiencia, compromiso, distanciamiento: un "hacer reflexivo" el lugar del cuerpo en la Educación Física. En: Adrián Scribano, Policromía corporal. Cuerpos, Grafías y Sociedad. Córdoba: Jorge Sarmiento Editor.

Landa, M.I. (2008). Proyecto de Investigación: El cuerpo del trabajo en las empresas de Fitness contemporáneas: Prácticas y subjetividades trasnacionales. CONICET. Doctorado en Ciencias Sociales. Universidad Nacional de Buenos Aires, Argentina.

Landa, M. I. (2009a). El porvenir de los cuerpos rentables: un análisis de la práctica del Fitness. En: R. Crisorio y M. Giles, Estudios Críticos de la Educación Física, (pp. 177-192). La Plata: Editoriales Al Margen.

Landa, M.I. (2009b). El porvenir de los cuerpos rentables: un análisis del dispositivo cultural del Fitness en Argentina. Tesis del Máster en Literatura comparada: estudios literarios y culturales. Barcelona: Universidad Autónoma de Barcelona, España. Inédito.

Landa, M. I. (2009c). Subjetividades y consumos corporales: un análisis de la práctica del Fitness. En dossier: "Deporte, Cultura y Comunicación", Razón y Palabra, Núm. 69. Disponible en: http://www.razonypalabra.org.mx/sobrerp.html. 
Landa, M.I. (2009d.). Os corpos da liderança: as tramas da ficção do dispositivo cultural do Fitness. En P. Gomes Dornelles e I. Wenetz (ed.), Educação Física, Corpo e Gênero: diálogos contemporâneos, dossier, Cadernos Cedes. Editorial: Universidade Estadual de Campinas (UNICAMP), Campinas (Brazil). En proceso de edición.

Landa, M. y Marengo, L. (2007a). "Empresa, Cuerpo, Vida, Tiempo Global: un análisis de la regulación de las energías corporales en las sociedades capitalistas flexibles". Ponencia presentada en el Séptimo Congreso Latinoamericano de Educación Física y $2^{\circ}$ Latinoamericano de Educación Física y Ciencia. La Plata: Facultad de Humanidades y Ciencias de la Educación. Universidad Nacional de La Plata, Argentina. Inédito.

Landa, M. y Marengo, L. (2007b). "Trabajo, Cuerpos y Emociones: de brazos entrenados a mentes gestionantes". Ponencia presentada en el XXVI Congreso de la Asociación Latinoamericana de Sociología. Guadalajara: Universidad de Guadalajara, Méjico. Publicación en Actas de Congreso (Formato CD).

Landa, M. y Marengo, M. (2009a). "Biopolíticas empresariales en la Argentina Contemporánea: Usos y articulaciones del "Fitness" en contextos productivos emergentes". Ponencia presentada en el Congreso Transdisciplinar sobre el cuerpo. El cuerpo: objeto y sujeto de las ciencias humanas y sociales. Barcelona. Institución Milá. CSIC, España. Publicación en Actas de Congreso.

Landa, M. y Marengo, L. (2009b). "Perfomances empresariales: el cuerpo de un líder", VIII Reunión de Antropología del MERCOSUR. Diversidad y Poder en América Latina, Universidad Nacional de San Martín, Buenos Aires, Argentina. Publicación en Actas de Congreso (Formato CD).

Landa, M. y Marengo, L. (2010a). La metabolización del cuerpo en el capitalismo avanzado, Trabajo y Sociedad. Número 14, Vol. XIII, Verano 2010. Disponible en: http://www.unse.edu.ar/trabajoysociedad/14_LANDA_MARENGO_Metabolizaci on_Cuerpos.pdf.

Landa, M. I. y Marengo, L. (2010b). Devenir cuerpo empresa: el nuevo capitalismo y sus tramas de sujeción. En dossier: "Cuerpos contemporáneos: nuevas prácticas, antiguos retos, otras pasiones", Actuel Marx, Núm.9. En proceso de edición.

Landa, M. y Sorribas, P. (2006). "El disciplinamiento corporal en las formas de organización del trabajo capitalistas" Ponencia presentada en Jornadas Preparatorias del XXVI CONGRESO ALAS de Guadalajara 2007, Foro Las morfologías del trabajo y la educación en la actualidad, Universidad Nacional de Cuyo Facultad de Ciencias Políticas y Sociales, Mendoza, Argentina.

Le Breton, D. (2002). La sociología del cuerpo. Buenos Aires: Ediciones Nueva Visión. 
Linhart, D. (1997). La modernización en las empresas. Buenos Aires: Asoc. Trabajo y Sociedad.

Marengo, L. (2007). Proyecto de Investigación: "La producción discursiva y los dispositivos de management en la nueva empresa. El fenómeno de los Call Centers en Córdoba, Argentina". CONICET. Doctorado en Semiótica. Centro de Estudios Avanzados. Universidad Nacional de Córdoba, Argentina.

Marengo, L. (2008a). "Representaciones sociales del trabajo de Call Centers. Un estudio de caso en la empresa Movistar". Ponencia presentada en las $V$ Jornadas de Sociología, Universidad Nacional de La Plata, La Plata, Argentina.

Marengo L. (2008b). "Discursos empresariales y prácticas de management en Call Centers. Comunidades imaginadas en las fábricas del habla". Ponencia presentada en II Congreso de la Sociedad Colombiana de Estudios Semióticos y de Comunicación, Bogotá, Colombia. Disponible en: http://socesc.googlepages.com/CSDiscursosempresarialesypracticasde.pdf

Marengo, L. (2009). "Metabolización Proteica en el capitalismo flexible", En: Biset, E., Lorio, N., Ricca,G. (ed.), Animales, hombres, máquinas: I Coloquio Nacional de Filosofia (pp 69-81), Universidad Nacional de Rio Cuarto, Rio Cuarto, Argentina.

Marx, K. (1973) "El capital: Critica de la economía política” Tomo I. Buenos Aires: SXXI.

Mauss, M. (1971). El concepto de técnica corporal. En Sociología y Antropología (pp 337-343), Madrid: Técnos.

Mellor, P. y Shilling, C. (1997). Re-forming the Body. Religion, Community and Modernity. Londres: Sage Publications Ltd.

Métzsáros, I. (1995). Más allá del capital: Hacia una teoría de la transición. Caracas:Vadel.

Pedraza, Z. (2003). Cuerpo e investigación en teoría social. Conferencia presentada en la Universidad de Colombia. Disponible en:. http://antropologia.uniandes.edu.co/zpedraza/zp1.pdf

Pedraza, Z. (2009). Derivas estéticas del cuerpo. Desacatos, Núm. 30, Agosto. Disponible en: http://www.scielo.org.mx/scielo.php?script=sci_arttext\&pid=S140592742009000200006\&lng $=$ pt\&nrm $=$ iso $>$.

Pierbasttisti, D. (2008). La privatización de los cuerpos. La construcción de la proactividad neoliberal en el ámbito de las telecomunicaciones, 1991-2001. Buenos Aires: Prometeo Libros.

Schilling, C. (1993). The Body and Social Theory. Londres: Sage Publications. 
Schilling, C. (1997). The undersocialised conception of the embodied agent in modern sociology, Sociology, Vol. 31, Núm.4, 737-54.

Sennett, R. (1998). La corrosión del carácter. Las consecuencias personales del trabajo en el nuevo capitalismo. Barcelona: Anagrama.

Soley-Beltran, P. (2007). Una introducción a la sociología del cuerpo. En M. Torrás (ed.), Cuerpo e Identidad I: Estudios de género y sexualidad 1(pp 247 - 265). Barcelona: Ediciones UAB.

Turner, B.S. (1992). The Body and Society. Explorations in Social Theory. London: Sage Publications.

Virno, P. (2008). Gramática de la Multitud: para un análisis de las formas de vidas contemporáneas. Buenos Aires: Colihue.

Wittke, T. (2006). "La empresa: nuevos modos de subjetivación en la organización del trabajo". En L. Schvarstein y L. Leopold, L. (Coor.) Trabajo y subjetividad. Entre lo existente y lo necesario (pp. 147-164). Buenos Aires: Paidos. 